\title{
Statistical Fluid Dynamics: Unstable Fingers
}

\author{
J. Glimm ${ }^{1 \star}$, D. Marchesin ${ }^{1 \star}$, and O. McBryan ${ }^{2 \star}$ \\ 1 The Rockefeller University, New York, NY 10021 and ${ }^{2}$ Courant Institute, New York University, \\ New York, NY 10012, USA
}

\begin{abstract}
This paper is the first in a series by the authors devoted to the study of fingers in fluid surfaces. Fingers are a form of surface instability which occur on many length scales. In particular, they may occur on length scales small relative to the natural dimensions of the problem; in this sense the instability is similar to turbulance. In the present study, the transition from stability to instability is determined by a critical value in a viscosity ratio. This series of papers is devoted to methods of accurate numerical computation. We find that the random choice method gives excellent resolution of fingered surfaces and discontinuities. Even an unstable interface, with three to four well developed fingers can be resolved on a coarse grid of 10 to 15 zones wide.
\end{abstract}

\section{Introduction}

Fingers are a form of surface instability; they occur in a number of physical phenomena. They often occur with a characteristic length (finger width, or finger spacing) which is small relative to the natural dimensions of the problem. In fact, fully developed fingering is approximately scale invariant, in that it may occur on all length scales from the dimensions of the problem down to a cutoff length provided by some dissipative mechanism.

We study this phenomenon numerically in the context of two phase flow in porous media.

The equations of two phase immiscible flow in porous media have the form

$$
\begin{aligned}
& \frac{\partial s}{\partial t}+\nabla \cdot(\mathbf{v} f(s)=0, \quad \mathbf{v}=-k(s) \nabla p, \\
& \nabla \cdot \mathbf{v}=0,
\end{aligned}
$$

* J.G. was supported in part by the NSF Grant PHY-78-08066; J.G. and D.M. were supported in part by the Army Research Office, ARO Grant DAAG 29-78-G-0171; O.M. was supported in part by the NSF Grant DMR 77-04105 USA

Permanent address of O.M.: Mathematics Department, Cornell University, Ithaca, NY 14853.

Permanent address of D.M.: Mathematics Department, Pontificia Universidade Catolica do Rio de Janeiro, Rio de Janeiro, Brazil 
neglecting capillary pressure (the dissipative mechanism). Here $s$ denotes the saturation (fraction of water in total fluid) and $p$ is pressure. Also $k$ and $f$ are known functions which describe the permeability and porosity of the reservoir and the viscosities of the two incompressible phases flowing in it. We use the functions

$$
\begin{aligned}
& k(s)=s^{2}+(1-s)^{2} / \mu, \\
& f(s)=s^{2} / k(s),
\end{aligned}
$$

where $\mu$ is the viscosity ratio for the fluids in question. In order to eliminate geometrical effects from the study of viscous fingering, we have chosen as a model geometry a rectangle $0 \leqq x \leqq X, 0 \leqq y \leqq Y$, and with the boundary conditions

$$
\begin{aligned}
& s(x, y=0, t)=1, \\
& \partial_{x} p(x=0, y, t)=\partial_{x} p(x=X, y, t)=0, \\
& p(x, y=0, t)=0, \quad p(x, y=Y, t)=v Y .
\end{aligned}
$$

In particular the role of the source terms normally present in the Eqs. (1.1) and (1.2) is played by the boundary data.

The problem (1.1)-(1.5) is scale invariant. This means that for any $a>0$,

$$
\begin{aligned}
& s^{\prime}(x, t)=s(a x, a t), \\
& p^{\prime}(x, t)=a^{-1} p(a x, a t),
\end{aligned}
$$

solves (1.1)-(1.5) in the rectangle $0 \leqq x \leqq X^{\prime}=a^{-1} X, 0 \leqq y \leqq Y^{\prime}=a^{-1} Y$ if $s, p$ solves $(1.1)-(1.5)$ in $[0, X] \times[0, Y]$. We have fixed $v=1$ throughout this paper. This is no loss of generality because change of $v$ is equivalent to change of time scale.

The problem we have addressed is to determine the oil-water saturation front, for values of $\mu$ corresponding to both stable and unstable (fingering) regimes. The answer should be independent of (a) mesh refinement, (b) choice of random number generator, (c) scale transformations (1.6), and (d) the value of the eccentricity ratio $e=X / Y$, for $X / Y$ not too small. (Our computations include values of $e$ as small as $e=1 / 4)$. In the unstable case, the front $y(x, t)$ is subject to random disturbances, which means that $y$ cannot be computed to meet criteria (a)-(d). Instead, we extract statistical information, namely the mean and variance, and find that these quantities are deterministic in the sense that computations of the mean and variance satisfy criteria (a)-(d). In the language of statistical mechanics, the mean and variance are thermodynamic functions of the ensemble.

The independence of eccentricity means that $x$-direction boundary effects have been eliminated. Conceptually, the problem can then be thought of as defined on an infinite strip $x \in R, y \in[0, Y]$. The problem is then $x$-translation invariant, and the transition to instability and formation of fingers is a symmetry breaking transition. By general principles the breaking of symmetry has the multiplicity of $G / G_{0}$ if $G$ is the full symmetry group and $G_{0}$ is the unbroken symmetry subgroup $\left(G=R, G_{0}=0, G / G_{0}=R\right.$ in our case). In the unstable case, only $x$-translation invariant functionals have values which are independent of exactly how the symmetry is broken.

Since the exact nature of the symmetry breaking is not part of the formulation of the problem, it is only these translation invariant functions which can be 
computed with an answer independent of mesh refinement and other accidental features of the computer code. We demonstrate numerical convergence for some specific translation invariant functionals chosen to give information about the oilwater front.

The second paper in these series introduces wells (point sources and sinks) into the flow field. The third paper elaborates on some numerical analysis aspects of the computations.

The problem of computing unstable fingering was previously considered by Peaceman and Rachford [11]. A general reference to petroleum reservoir problems is [12]. The computer programs related to this paper were written in the $\mathrm{C}$ programming language and were developed on the UNIX Operating System.

\section{The Numerical Methods}

The random choice method $[6,2]$ was first used to solve the Buckley-Leverett equation $(1.1)$ in $[1,4])$. The random choice method $[6]$ uses the physically correct structure of waves and their interactions combined with a statistical sampling procedure. The method is characterized by first order accuracy (when applied to typical problems on practical mesh sizes) and exceedingly good resolution. The method makes use of a sequence $\left\{\theta_{n}\right\}$ of numbers [here we choose $\theta_{n}$ to be the fractional part of $(n+$ const $) \sqrt{2}]$ equidistributed on the interval $[0,1]$. In this method of solution, each wave in the solution achieves its correct speed statistically (as in a random walk), but because adjacent fluid blocks are never averaged or mixed, numerical diffusion is completely eliminated. A detailed discussion of this method can be found e.g. in [3]. The extension of the one dimensional random choice method [6] to higher dimensions is by operator splitting $[2,14]$ (a numerical version of the Trotter product formula), in which the successive solution of one dimensional $(x, t)$ and $(y, t)$ problems approximates the solution of a two dimensional $(x, y, t)$.

The problem we are solving has no $x$-dependence, so its mathematical solution is independent of $x$ and can be found in closed form by the method of lines. However this solution is unstable for $\mu$ sufficiently large, and the solutions of physical interest are those which result from small ( $x$-dependent) perturbations of the data. Here the randomness of the random choice method is an advantage, because it introduces small random perturbations into the solution at each time step. In the stable region, $\mu<\mu_{\text {crit }}$ these small perturbations are damped out and do not affect the solution, while in the unstable region, $\mu>\mu_{\text {crit }}$, the small perturbations grow to produce fingers and occasional isolated phase islands: oil surrounded by water or vice-versa. In this sense, the random choice method can be thought of as simulating a slightly heterogeneous medium. In the present paper, we have no control over the degree of heterogeneity except that we choose distinct random number generators for each column $x=$ const in the mesh. This is achieved by choosing the constant in

$$
\theta_{n} \equiv(n+\text { const }) \sqrt{2}(\bmod 1)
$$

to depend on $y$. 


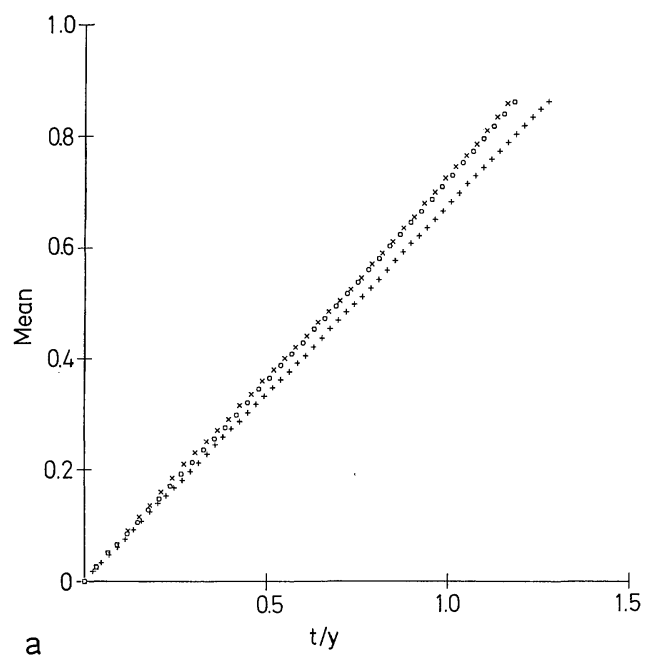

Fig. 1a. The mean position of the oil water interface for oil water viscosity ratio $\mu=\mu_{o} / \mu_{w}=2$. Two runs are presented, each with $20 y$-mesh blocks. $x=10 x$-mesh blocks and $\square=20 x$-mesh blocks. For comparison, the mean of an ensemble of one dimensional problems, calculated by the same procedure is shown with the legend +

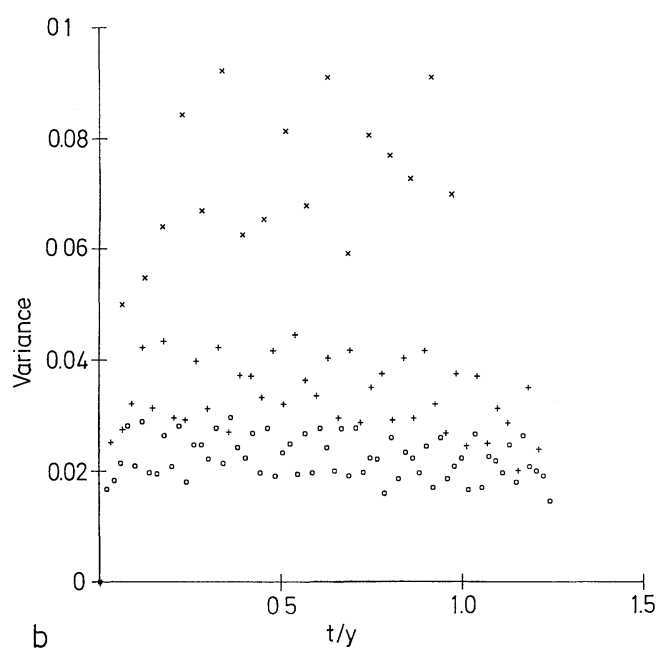

Fig. 1b. The variance of the oil water interface for oil water viscosity ratio $\mu=\mu_{o} / \mu_{w}=2$. This is the stable case and the variance does not increase with time. Three runs are presented here, all have 20 $x$-mesh blocks. $x=10 y$-mesh blocks, $+=20 y$-mesh blocks, and $\square=30 y$-mesh blocks

To reduce the heterogeneity, we propose tracking of the front, cf. [7], and to increase heterogeneity, one could take $k(s)=k(s, x, y)$ to be a random field with a lognormal distribution of permeabilities, cf. $[5,9]$. Heterogeneity is also reduced by $y$-direction mesh refinement (see Fig. 1b) because the statistics generate fluctuations on the level of one $y$-mesh block. 
The elliptic equation was solved by an accelerated [8] version of the conjugate gradient [10] algorithm. Because of the sharp resolution in the solution of the hyperbolic equation, the elliptic problem has discontinuous coefficients; moreover for $\mu>\mu_{\text {crit }}$, the discontinuity surface is highly irregular. We used a finite element method [13] with piecewise linear elements. The exact solution of the pressure equation is continuous but only piecewise smooth, so the elements had no more regularity than the exact solution.

It is a pleausre to thank O. Widlund for helpful discussions on the elliptic acceleration.

\section{The Results}

A) $\mu=2$

For $\mu=2$ the oil water surface is stable. The computed interface

$$
y_{\text {front }}=y(x, t),
$$

has fluctuations which go to zero with $\Delta y$. In the dimensionless variables

$$
\begin{aligned}
& \tau=t / Y, \\
& \psi(x, \tau)=y_{\text {front }}(x, \tau Y) / Y,
\end{aligned}
$$

we define the mean and variance

$$
\begin{aligned}
& m(\tau)=X^{-1} \int_{0}^{X} \psi(x, \tau) d x, \\
& v(\tau)=X^{-1 / 2}\left(\int_{0}^{X}(\psi(x, \tau)-m(\tau))^{2} d x\right)^{1 / 2} .
\end{aligned}
$$

scaled to be independent of $X$ and $Y$.

Because the interface is stable, $v(\tau)$ is bounded in $\tau$. A scaling analysis suggests $v(\tau) \sim \Delta y / Y$. For comparison, we computed a one dimensional problem corresponding to (1.1)-(1.5) with different random number generators. The result produced the same $m$ and $v$, within the accuracy of the computation. Thus it appears that the statistical fluctuations from the random number generator and the nonlinear dynamics from the Buckley-Leverett equation are not interacting with each other. In Fig. $1, m(\tau)$ is plotted for two levels of $\Delta$ mesh refinement and for the decoupled ensemble of independent one dimensional problems; $v(\tau)$ is plotted for three levels of $\Delta y$ mesh refinement.

\section{B) $\mu=4$}

This value of $\mu$ marks the beginning of instability. The fingers form and grow slowly, so that by eye, they are hard to distinguish from the statistical fluctuations introduced by the random number generator. However, the plot of $v(\tau)$ for two levels of mesh refinement shows clearly that the fingers are growing linearly in time (see Fig. 2b). Figure 2a is the plot for $m(\tau)$. Note the good agreement obtained even for a very coarse mesh. 


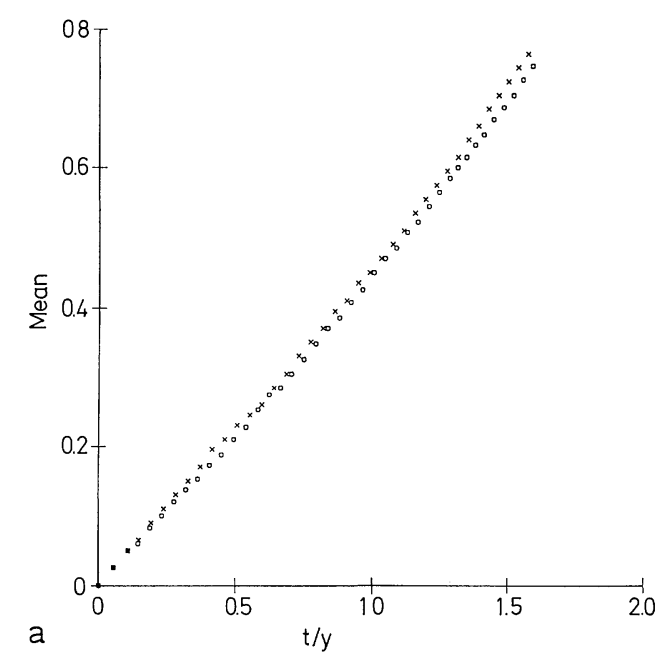

Fig. 2a. The mean position of the oil water interface for oil water viscosity ratio $\mu_{o} / \mu_{w}=4$. Two runs are presented, each with $20 y$-mesh blocks. $x=10 x$-mesh blocks and $\square=20 x$-mesh blocks

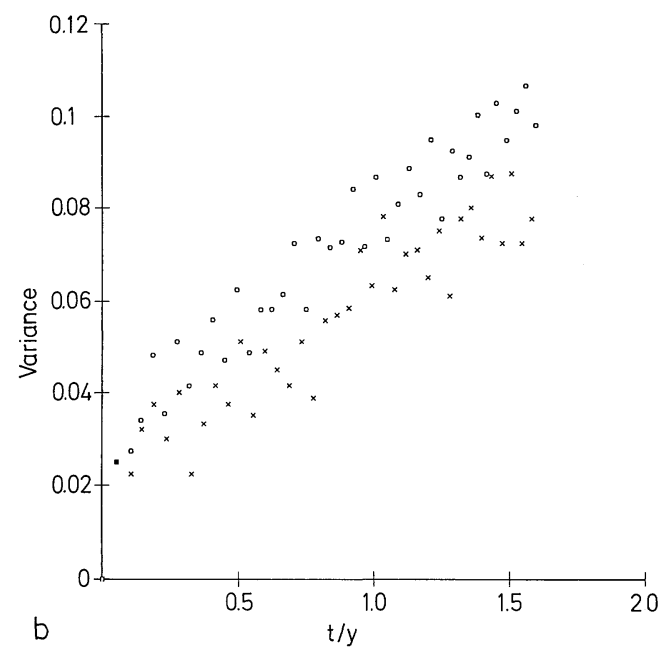

Fig. 2b. The variance of the oil water interface for oil water viscosity ratio $\mu=\mu_{o} / \mu_{w}=4$. This is the unstable case. At $t / y=0$ the variance begins at a nonzero level due to numerical heterogeneity effects. The linear increase with time is due to the dynamical instability. Two runs are presented, with 20 $y$-mesh blocks. $x=10 x$-mesh blocks and $\square=20 x$-mesh blocks

C) $\mu=10$

The instability of the fingers is now apparent, and a new phenomena appears: $y_{\text {front }}$ is no longer singlevalued. To resolve this ambiguity, we choose a saturation contour level line as the first approximation to (3.1). Because of the sharp resolution, the advancing saturation front has a dominant value $s_{f}$, and we choose the level line corresponding to $s_{f} / 2$ to define the first approximation to $y_{\text {front }}$. This level line occasionally fails to be single valued as a function of $x$; we then choose 


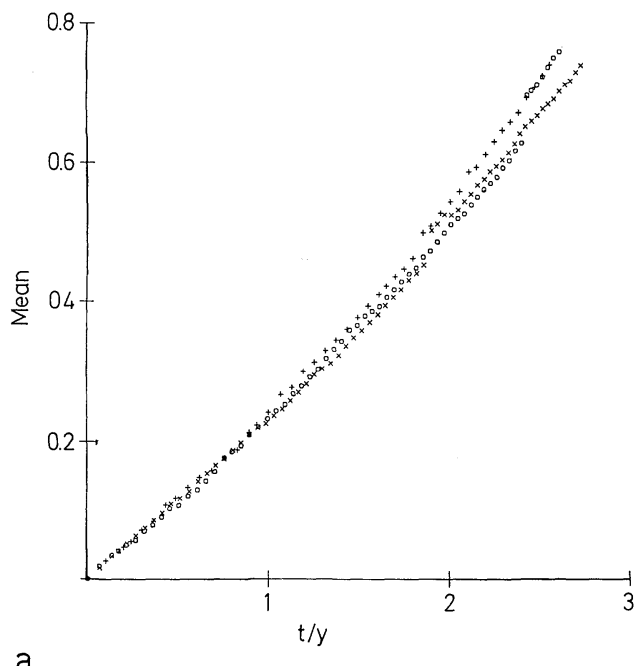

a

Fig. 3a. The mean position of the oil water interface for oil water visocisty ratio $\mu_{o} / \mu_{w}=10$. Three runs are presented, with eccentricity ratios $e=1(x) e=1 / 2(\square)$ and $e=1 / 4(+)$. The three runs also used distinct random number generators and mesh spacing

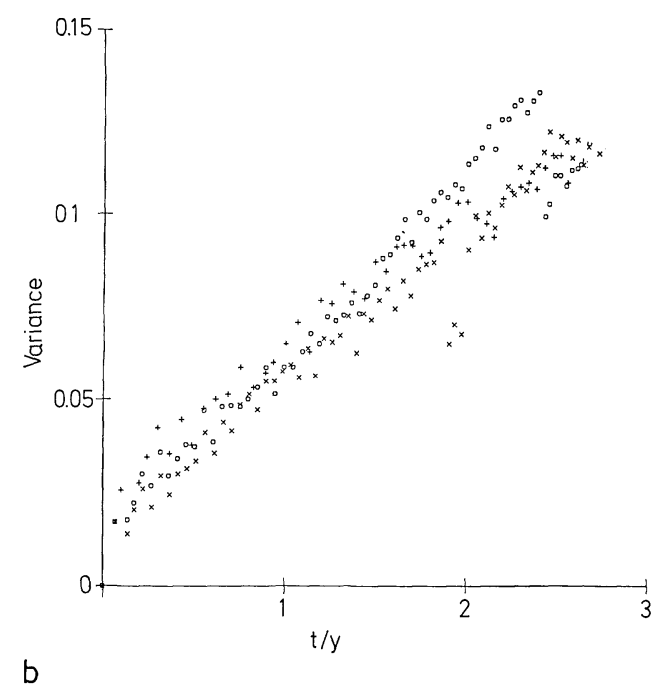

Fig. 3b. The variance of the oil water interface for oil water viscosity ratio $\mu_{o} / \mu_{w}=10$. The same runs as in Fig. 3a are presented here

the larger branch. With this choice of $y_{\text {front }}$, we plot $m(\tau)$ and $v(\tau)$ for several choices of mesh level, eccentricity and random number generator. The results are in good agreement (see Fig. 3a and b). Even a very coarse mesh $(10 \times 20)$ gave good agreement for $m(\tau)$, and for $\tau$ up to $3 / 4 \tau_{\text {breakthrough }}$ for $v(\tau)$.

In Fig. 3c, we give saturation values for a $30 \times 30$ mesh. Qualitatively, one sees the same picture on a $10 \times 20$ mesh. The resolution at the edge of the front is nearly 
Time Data: $42 \mathrm{nd}$ Time Step, Time $=1.91694$, Time Step $=0.037618$

Grid Data: $X=1, Y=1, \Delta x=0.0333, \Delta y=0.0333$

Saturation: (A's removed)

$\operatorname{Max}=1 \mathrm{Min}=0$

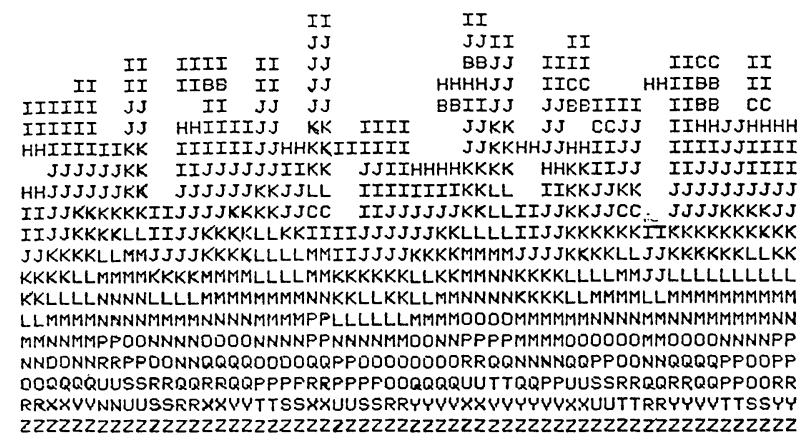

Fig. 3c. Saturation values for oil water viscosity ratio $\mu_{o} / \mu_{w}=10$. Each mesh block is a 2 letters wide and one letter high. The saturation values are plotted on an alphabetic scale, so that $Z Z$ represents saturation in the interval $[25 / 26,1]$. $A$ 's have been removed, and the sharp discontinuity in the front is seen by the jump from $H$ 's or I's to A's (removed) along most of the front

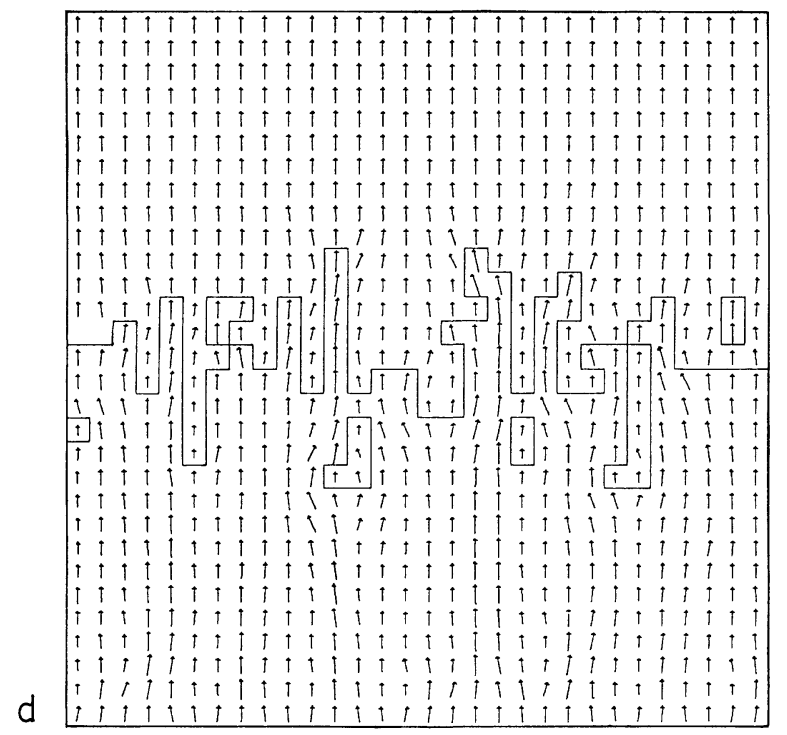

Fig. 3d. Velocity field and saturation contour level line for saturation value $s=5 / 26$, and the same run and time step as Fig. 3c. $x$ components of the velocity field have been magnified by 5

perfect. Velocity plots are shown in Fig. 3d, for the same time step, for the $30 \times 30$ mesh. For visual clarity, the $x$ velocity component is multiplied by five relative to the $y$ velocity component. Note the tendency of the velocity to avoid the trench - i.e. inverse or oil finger, and to expand at the tip of the water finger. In the stable case $(\mu=2)$ these tendencies are reversed. 


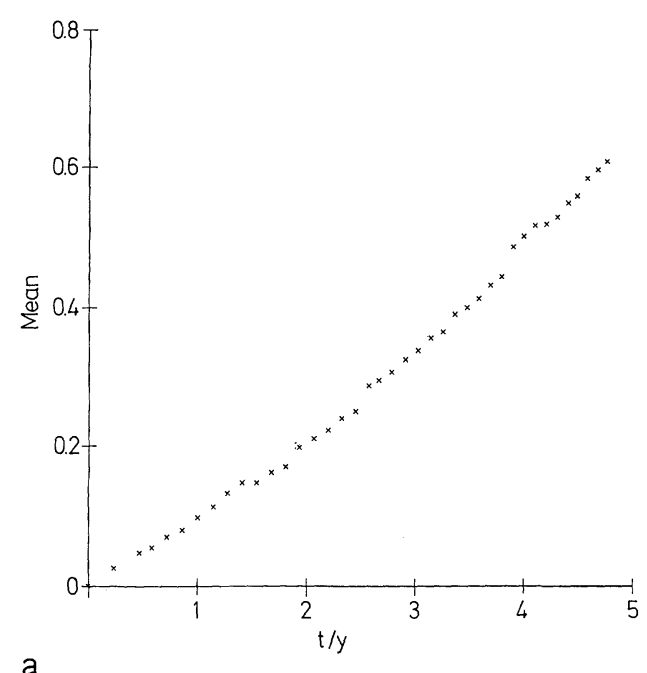

a

Fig. 4a. $m(\tau)$ vs $\tau$ for $\mu=40$

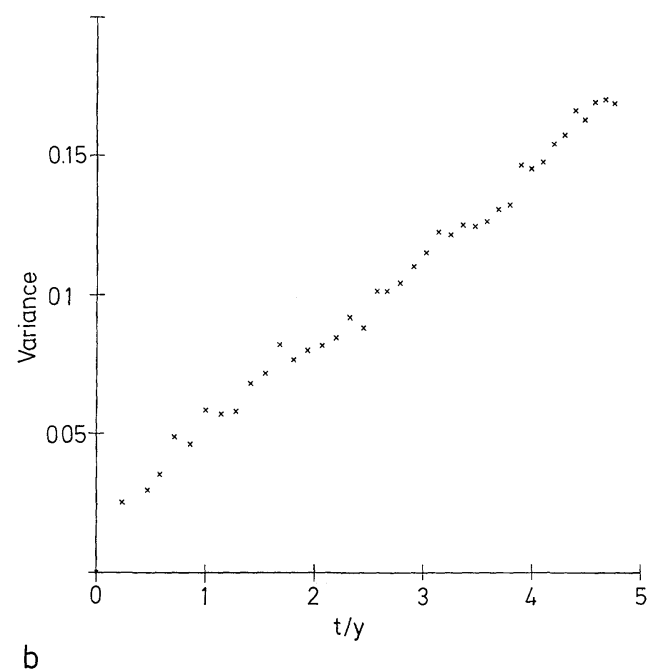

Fig. 4b. $v(\tau)$ vs $\tau$ for $\mu=40$

D) $\mu=40$ and $\mu=80$

Here the loss of single valuedness of $y_{\text {front }}$ becomes fairly marked, and occasional phase islands appear (oil surrounded by water or vice-versa). The case of isolated water droplets seem to be caused by numerical effects; they are probably connected to the front by a narrow stream, not resolved on this mesh spacing. Plots for $m(\tau)$ and $v(\tau)$ are given in Figs. $4 \mathrm{a}$ and $\mathrm{b}$ and $5 \mathrm{a}$ and $\mathrm{b}$ while saturation levels are given in Figs. $4 \mathrm{c}$ and $5 \mathrm{c}$. Figures $4 \mathrm{~d}$ and $5 \mathrm{~d}$ give velocity plots, with $v_{x}$ magnified by a factor of 5 . 
Time Data: 40 th Time Step, Time $=9.71944$, Time Step $=0.146781$

Grid Data: $X=1, Y=2, \Delta x=0.05, \Delta y=0.1$

Saturation: ( $A$ 's removed)

$\operatorname{Min}=1 \mathrm{Min}=0$

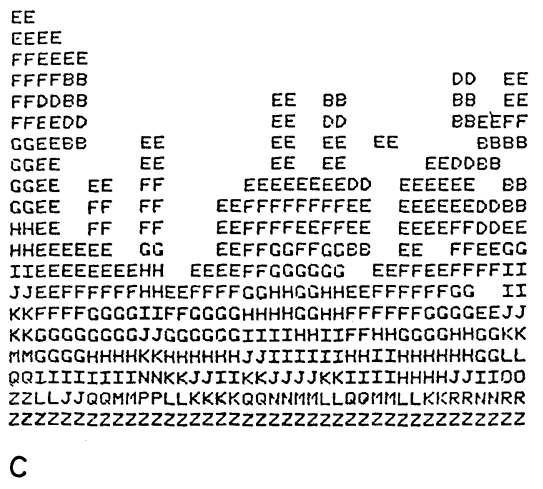

Fig. 4c. Saturation values, on an $A-Z$ scale, for $\mu=40$, on a $20 \times 20$ grid

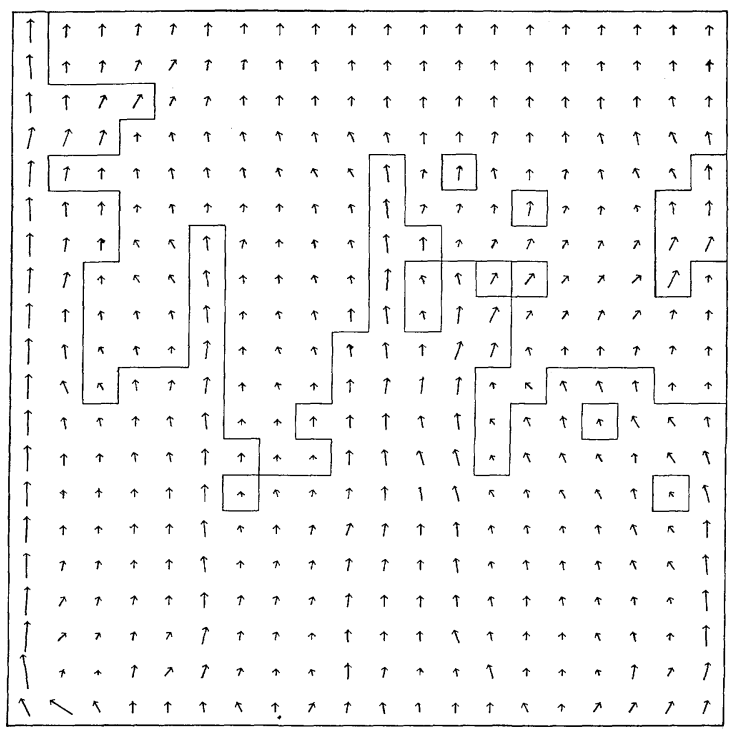

d

Fig. 4d. Velocity values (exaggerated by a factor of 5) for $\mu=40$, for the same run and time as Fig. 4c. Note $X=1, Y=2$ 


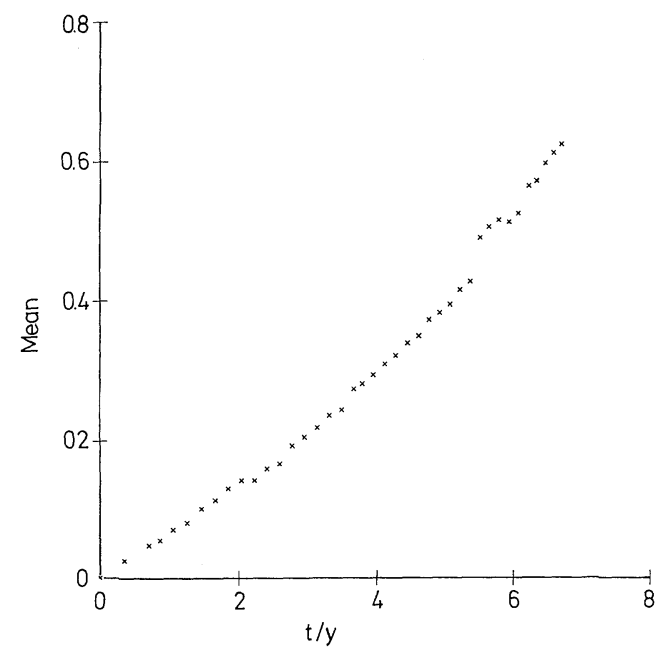

a

Fig. 5a. $m(\tau)$ vs $\tau$ for $\mu=80$

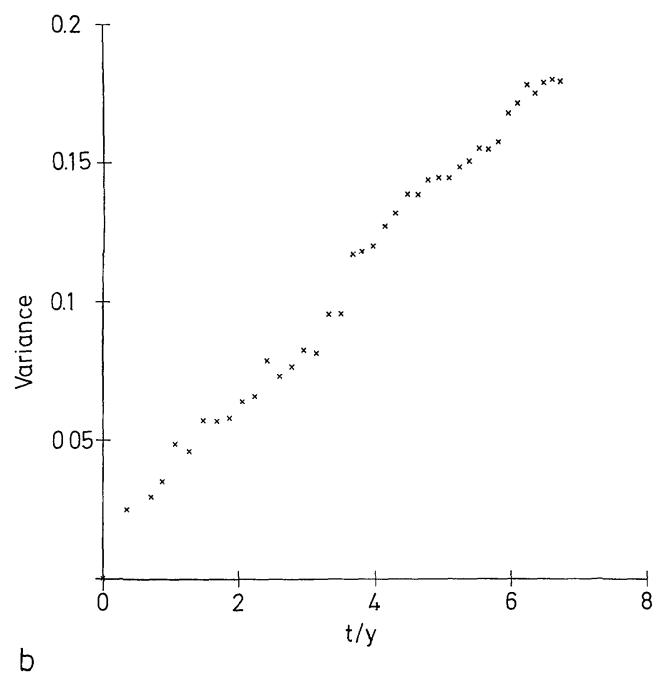

Fig. 5b. $v(\tau)$ vs $\tau$ for $\mu=80$ 
Time Data: 40th Time Step, Time $=13.7156$, Time Step $=0.204564$

Grid Data: $X=1, Y=2, \Delta x=0.05, \Delta y=0.1$

Saturation: ( $A$ 's removed)

$\operatorname{Max}=1 \mathrm{Min}=0$

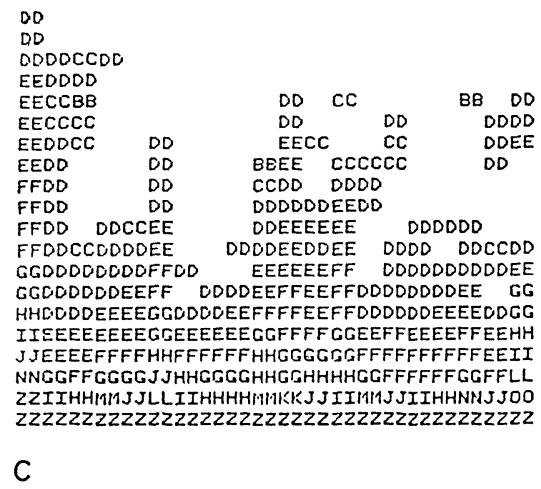

Fig. 5c. Saturation values, on an $A-Z$ scale, for $\mu=80$, on a $20 \times 20$ grid

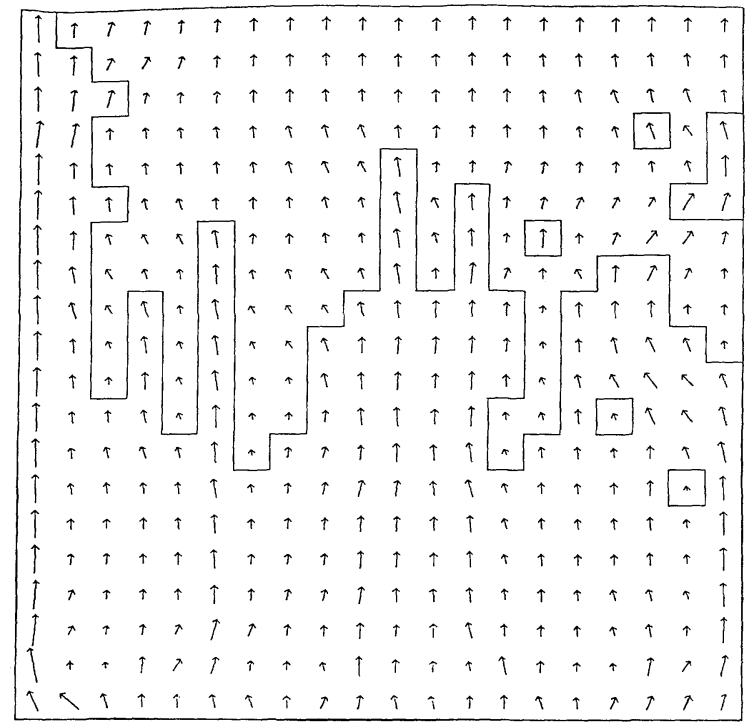

d

Fig. 5d. Velocity values ( $v_{x}$ exaggerated by a factor of 5 ) for $\mu=80$, for the same run and time as Fig. 5c. Note $X=1, Y=2$

\section{Conclusions}

We have seen that the random choice method can resolve sharply moderately complex wavefronts in a two dimensional problem even on a coarse grid. The resolution was aided by the fact that the wavefronts were dominantly parallel to the grid geometry. In addition to numerical grid effects, the phenomena of 
fingering itself is strongly affected by the curved geometry introduced by the flow patterns around producing and injecting wells.

\section{References}

1. Albright, N., Concus, P., Proskurowski, W.: Numerical solution of the multidimensional BuckleyLeverett equation by a sampling method. Presented at the Society of Petroleum Engineers 5th Symposium on Reservoir Simulation, Denver, Colorado 1979

2. Chorin, A.: Random choice solutions of hyperbolic systems. J. Comp. Phys. 22, 517 (1976)

3. Collela, P.: An analysis of the effect of operator splitting and of the sampling procedure on the accuracy of Glimm's method. University of California Thesis, Berkeley 1979

4. Concus, P., Proskurowski, W.: Numerical solution of a nonlinear hyperbolic equation by a random choice method. J. Comp. Phys. (to appear)

5. Dykstra, H., Parsons, R.: The prediction of oil recovery by water flood. In : Secondary recovery of oil in the United States, 2nd. ed, p. 160. API 1950

6. Glimm, J.: Solutions in the large for nonlinear hyperbolic systems of equations. Commun. Pure Appl. Math. 18, 697 (1965)

7. Glimm, J., Marchesin, D., McBryan, O.: Subgrid resolution of fluid discontinuities. II (preprint)

8. Hayes, Young: The accelerated SSOR method for solving large linear systems. Preliminary report. University of Texas at Austin, 1977

9. Law, J.: Statistical approach to the interstitial heterogeneity of sand reservoirs. Trans. AIME 155, $202(1944)$

10. Page. Sandus: Solution of space indefinite systems of linear equations, SIAM J. Num. Anal. 12, 617 (1975)

11. Peaceman, D., Rachford, H.: Numerical calculation of multidimensional miscible displacement. J. Soc. Pet. Eng. Dec. 1962, p. 327

12. Peaceman, D.: Fundamentals of numerical reservoir simulation. Amsterdam, Oxford, New York: Elsevier 1977

13. Strang, W.G., Fix: An analysis of the finite element method. New York: Prentice Hall 1973

14. Strang, W.G.: On the construction and comparison of difference schemes. SIAM J. Num. Anal. 5 , $506(1698)$

Communicated by A. Jaffe

Received October 29, 1979 
